\title{
BOOSTING EFFECT OF CONCENTRATED GROWTH FACTOR ON OSSEOINTEGRATION OF IMMEDIATE IMPLANT: A HISTOLOGICAL ANALYSIS IN DOGS
}

\author{
Tarek Abd Elbary Abd Ellatyf Saad*
}

\begin{abstract}
Background: Implant osseointegration is an important of long term success of the widely used Rehabilitation treatment. Many therapeutic presidiums have been investigated to accelerate osseointegration. Concentrated growth factors are third generation platelet concentrate used clinically in acceleration of tissue healing and bone regeneration. The aim of this study was to assess histologically the effect of using CGF on osseointegration of immediate dental implant in animal model
\end{abstract}

Methods: ten animal models (adult mongrel dogs) were selected in this study each to receive one immediate implant of the Spectra system (Inc. Las Vegas, NV 89149, CA. USA). They were divided in to 2 equal groups; group A received immediate implant without CGF as a control group and group B received immediate implant with CGF which was applied to the socket walls before implant placement. Eight weeks postoperatively the animal models were euthanized and implants with surrounding bone were dissected and transferred in formalin to the lab for histologic preparation and histomorphometric analysis

Results: histomorphometric analysis revealed the mean percentage of new bone formation for group A (control group) was $25.662 \%$ while for group B it reached $66.939 \%$. Student's t -test revealed that the difference between both groups was statistically significant difference with $\mathrm{p}$ value of 0.0001 which indicated the higher amount of bone regeneration in group B indicative of the accelerated osseointegration process in the test group.

Conclusion it may be concluded that application of CGF to the socket walls before placement of immediate implant may enhance the healing of bone surrounding implants and accelerate osseointegration providing a convenient and affordable choice for implant placement. Application of this method may, therefore, be effective in enhancing the rate of osseointegration and allow for early loading of immediate implants.

\footnotetext{
* PHD Maxillofacial Department, Faculty of Oral and Dental Medicine, Cairo University
} 


\section{INTRODUCTION}

Despite the ongoing advances of modern dentistry; loss of natural teeth is still inevitable. For many people the loss of teeth can mean the loss of jaw function and can lead to poor oral aesthetics and severe emotional distress. During the last decade implantology has become an indispensable part of dentistry; allowing dentists to improve the quality of life of a large number of patients and have revolutionized the way by which missing teeth are replaced with a high success rate; it is now considered the treatment of choice ${ }^{1}$

A Dental implant is a prosthetic device placed into the oral tissues under the mucosal or/and periosteal layer, and on/or within the bone to offer retention and support for a removable or fixed dental prosthesis $^{2}$

Osseointegration of dental implants is important for long term success and stability and it all starts with Bone healing around implants which involve a series of cellular and extracellular biological events take place at the bone - implant interface for healing of bone around implants till the implant surface is covered with a newly formed bone In an osteogenic processes comparable to those of the bone healing process, in terms of initial host response, growth and differentiation factors released by the activated blood cells at the bone-implant interface which regulates this series of biological events. ${ }^{3}$

The first biological component to come into contact with an endosseous implant is Blood. Blood cells at the implant interface release cytokines and other soluble, growth and differentiation factors. Platelets undergo biochemical and morphological changes including adhesion, spreading, aggregation and intracellular biochemical changes. The formed fibrin matrix acts as an osteoconductive substance for the osteogenic cells to adhere to and start forming bone.

Osteogenic cells form osteoid tissue and new trabecular bone that finally remodels into lamellar bone in direct contact with most of the implant surface (osseointegration). ${ }^{4}$

Growth factors are proteins that regulate the processes of wound healing. Platelet derived growth factor (PDGF), Transforming growth factor $-\beta$ (TGF $-\beta)$, Fibroblastic Growth Factor (FGF), Insulin- like growth factor I and II (IGF I \& II), Bone morphogenes proteins (BMPs) are the factors mediates the healing process. Platelet concentrate such as platelet rich plasma (PRP), Platelet rich fibrin (PRF), and Concentrated growth factors (CGF) have been used to accelerate tissue healing for a long time. Among these concentrates, CGF that shows better regenerative capacity than PRP and PRF. ${ }^{5}$

Concentrated growth factor (CGF) is an autogenuous product formed from venous blood by selective centrifugation. Local growth factors in human platelets would enhance healing of grafts and also counteract resorption. In 2006, Sacco introduced concentrated growth factors (CGFs), which contain more growth factors and a more rigid fibrin structure. Therefore, CGFs have shown more effective bone formation or soft tissue healing. ${ }^{6}$

Since then many studies have been conducted to investigate the possible benefits from applying CGF in the field of oral and maxillofacial surgery. Honda etal $2013{ }^{7}$ Found that CGF promoted proliferation, osteogenic maturation and mineralization of mesenchymal stem cells in vitro more over the authors found that combining CGFs with bone marrow stroma cells resulted in excellent healing of critical sized bone defects in vivo.

Chen etal $2016{ }^{8}$ Evaluated short term performance of implants placed after modified osteotome sinus floor elevation with CGFs in atrophic maxilla. The results showed high survival rate for these cases.

Durmuslar etal $2016{ }^{9}$ Compared bone healing around implants when placed alone (control) and 
when placed with autologous bone and when combined with CGFs in animal model. The results came in favor of CGFs combined implants . Wang etal $2016{ }^{10}$ Tested the effect of CGFs in treatment of induced TMJ osteoarthritis in goats. Results revealed new cartilage and bone regeneration when CGF was used. The authors concluded that the use of CGF method can replace the chondrocyte scaffold approach in cartilage regeneration.

Pirpir etal $2017{ }^{11}$ Assessed the effect of CGF on implant stability and osseointegration in patients. The authors concluded that CGFs had positive effect on implant stabilization and accelerated osseointegration.

Bonazza etal $2018{ }^{12}$ Conducted an in-vitro study to investigate the effect of soluble form of silicon with or without CGF and CGF alone on 3 human cells (fibroblasts, endothelial cells and osteoblasts). Authors found that CGF stimulates cell growth, proliferation, and metabolic activity, of all 3 tested cells suggesting that this treatment could be effective in tissue regeneration

Talaat etal $2018{ }^{13}$ investigated the effect of CGF application after enucleation of pathologic lesions from mandible. Results revealed significantly higher bone density in cases treated with CGF; indicating that this line of treatment is a cost effective and safe biotechnology approach for bone regeneration in surgical bone defects.

Various strategies are being explored to improve the quality and accelerate the osseointegration of a dental implant one of which is the bioactive molecules as CGF that have proven to be valuable in increasing osteoblastic differentiation and healing acceleration. ${ }^{14}$

\section{AIM OF THE STUDY}

The aim of this study was to assess bone healing histologically around dental immediate implants with and without the application of concentrated growth factors in animal model.

\section{METHODES}

\section{Animal selection and preparation}

Ten Healthy male adult mongrel dogs, weighing $15 \mathrm{Kgs}$ to $20 \mathrm{Kgs}$ were selected to receive 10 immediate implants were used in the current study

All guidelines regarding the care of animal research subjects were strictly followed in this study; all captured dogs had a veterinary health care $\&$ were housed in the animal house of Faculty of Oral and Dental Medicine - Cairo University. They received all needed medication, kept in separate cages \& properly fed. The kennels were sprayed with Neocidal (diazinon) (Misrpharmaceutical, co, Egypt) in concentration 6/1000 $\mathrm{ml}$. All the animals were injected subcutaneously with Ivermectin (Ivomec) (,Misrpharmaceutical,co ,Egypt.) 0.1 $\mathrm{ml} / \mathrm{kg}$ body weight to guard against external and internal parasites.

\section{Grouping:}

The 10 selected animal models were equally divided into 2 groups group A and group B. Group A: five immediate implants were placed in fresh extraction socket of the anterior or premolar maxillary area (control group). Group B: five immediate implants were placed with concentrated growth factor (CGF) which was laid down into the fresh extraction socket of the anterior or premolar maxillary area (test group).

\section{Concentrated growth factors (CGF) preparation}

A standard, disposable, $10 \mathrm{ml}$ non-anticoagulant tube and a matching centrifuge device were used. Intravenous blood samples were drawn from the jugular vein and placed in centrifuge tube without anticoagulants. Then the tubes were placed in the centrifugation device in an opposing balanced manner and rotated in four sequential steps. The first step at $735 \mathrm{~g}(2249 \approx 2200)$ for $2 \mathrm{~min}$., the second one at $580 \mathrm{~g}(1998 \approx 2000)$ for $4 \mathrm{~min}$., the third one at $735 \mathrm{~g}(2249 \approx 2200)$ for $4 \mathrm{~min}$. and the fourth one 
at $905 \mathrm{~g}(2495 \approx 2500)$ for $3 \mathrm{~min}$. The result was a clot that was collected using a straight tweezer and ready to be used

\section{Surgical steps and procedures:}

Prior surgery dogs were given antibiotic therapy IM (cefoperazone sodium 40mgไKg). (Pfizer Egypt S.A.E).Twelve hours before the surgery food and water were stopped. Half an hour before surgery premedication in the form of atropine sulfate (Misr pharmaceutical Co. Egypt.) (0.5 $\mathrm{mg} / \mathrm{kg})$ body weight was administrated IM. Surgical procedure was made under general anesthesia with aseptic condition. Anesthesia: Thiopental sodium (Epico co, Egypt.) $30 \mathrm{mg} k \mathrm{~kg}$ body weight) in saline was injected IV. The surgical site was disinfected by Bovidone iodine $1 \%$.

In group A; Gingival (sulcular) mucoperiosteal incision was performed around the neck of the tooth to be extracted and extended around the mesial and distal adjacent teeth to allow exposure the field of surgery. The tooth was gently extracted as atraumatically as possible to preserve available bone following extraction particularly the labial and buccal wall.

The socket was prepared to receive the immediate implants of Spectra system (Inc. Las Vegas, NV 89149, CA. USA.), thoroughly debrided by careful curettage and irrigated with sterile saline solution to remove any infected or inflammatory tissues as well as remnants of the periodontal ligament. The implant bed was drilled at the bottom of the socket using the drilling sequence recommended by manufacturer.

All drilling cascades were completed using a high torque low speed surgical motor with an internal irrigation device. The drilling speed was $800 \mathrm{rpm}$, and was decreased to $650 \mathrm{rpm}$ in dense bone. Cooling system in the form of normal saline was utilized. The drilling was performed to reach the appropriate depth reference line. The drilling was continuing till engagement of the drill with the apical part of the socket was detected with increasing the depth 3-4 mms apically.

Drilling was performed to enlarge the implant site and increasing in size until the selected implant diameter was reached. The prepared sites then irrigated with normal saline to remove any bony fragments. After preparation of the implant site the implant was removed from the inner vial by the silicon stopper without breaking the sterile chain, guided into the cavity and inserted with finger pressure.

The stopper was removed then the remounted placement head was also removed from the implant. The implant driver was positioned in the internal hex and the implant was threaded in place with approximately three revolutions in clockwise direction into the final position using the ratchet. Once the implant has been seated, the implant driver was removed from the internal implant-abutment connection. The cover screw in the silicone stopper is removed and threaded into the implant hex driver.

In group B (CGF group); the surgical procedures were performed in the same manner, before the implant insertion, the socket walls were laid with CGF. Flaps were sutured with $3 \backslash 0$ silk suture material without covering the gingival formers .

\section{Post-operative care:}

Ten animals were kept for recovery under close supervision. The animals were given an antibiotic (Cefoperazone sodium $40 \mathrm{mg} \backslash \mathrm{Kg}$ and an ant inflammatory drugs Ordis (Avents, Egypt). 20mg $\backslash \mathrm{Kg}$ via intramuscular injection, two divided dose per day for 5 days postoperatively. All animals were consuming soft diet for the first week postoperatively. Follow- up of the surgical sites with regards to gingival health, maintenance of suture line closure, edema, and evidence of tissue necrosis or infection was recorded. 


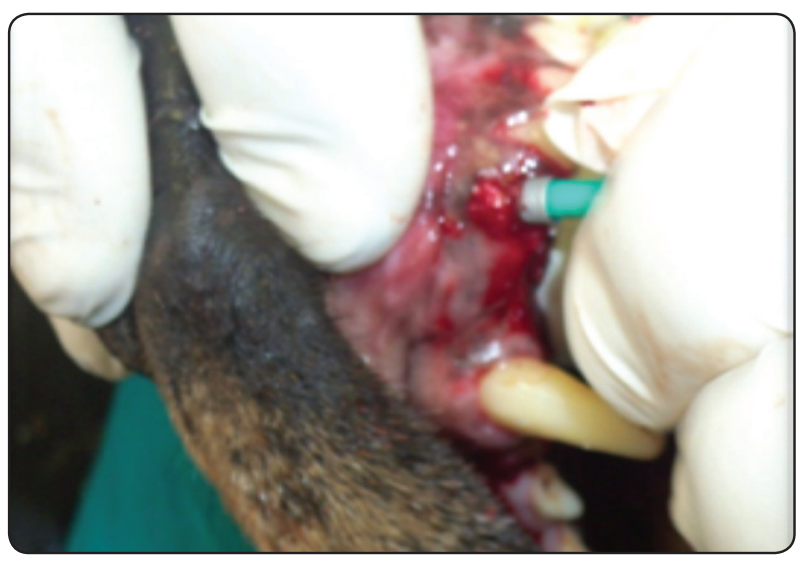

Fig. (1) Photograph showing immediate implant insertion through the socket of the right maxillary lateral incisor tooth, (Case no.1 of group A).

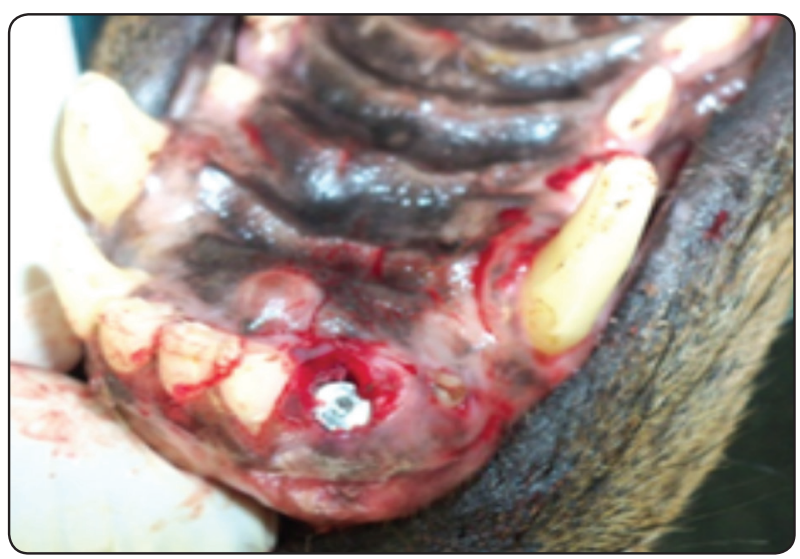

Fig. (2) Photograph showing the cover screw connected to the implant body installed through the socket of the right maxillary lateral incisor, (Case no.5of group B)

\section{Euthanasia:}

At the end of the experimental period ( 8 weeks post operatively) the animals were sacrificed. The animals were injected with $20 \mathrm{ml}$ Nesdonal solution; $5 \%$ rapidly injected through the cephalic vein. The implant with the surrounding bone were harvested for histological and histomorphometric evaluation.

\section{Follow up and postoperative Evaluation:}

All specimens were subjected to histological evaluation and histomorphometric analysis. Block section including the implant, surrounding bone has been taken. The implant sites was collected and sectioned for histological examination and histomorphometric evaluation.

\section{Histological preparation:}

Immediately after scarification, implants with the surrounding alveolar bone were dissected and biopsies were fixed in neutral buffered $10 \%$ formalin. Specimens were decalcified in $5 \%$ formic acid for 4-6 weeks. After adequate decalcification implants were gently removed from the specimens, and then tissues were dehydrated in ascending grades of ethyl alcohol and embedded in paraffin. Five $\mu \mathrm{m}$ thick sections were cut in a buccolingual direction. Tissue sections were cleared in xylene, rehydrated and stained by hematoxylin and eosin and examined by light microscopy.

\section{Histomorphometric analysis:}

H\&E stained sections were assessed by ordinary light microscope to detect the newly formed bone trabeculae. An image analyzer computer system software Leica* Quin500 (Leica Microsystems Inc., Switzerland) was used to process the images.

The percentage of the area of newly formed bone trabeculae was measured as area and area percent in a standard measuring frame of area 38883.69 micrometer ${ }^{2}$ per 10 fields in each. Mean values were obtained for each specimen.

\section{Statistical analysis:}

Data related to the histomorphometric analyses were presented as means and standard deviation (SD) values. Student's T-test was used to compare between means. The significance level was set at $\mathrm{P} \leq 0.05$. Statistical analysis was performed with SPSS** 16.0 (Statistical Package for Scientific Studies) for Windows.

\section{RESULTS:}

All implants successfully healed without any postoperative complications. No mortality, infection 
or wound exposure or change in body weight was noted. All the cases of the experimental animal models group (group B) were sacrificed 8 weeks postoperatively and prepared for microscopic examination.

Group A (Control group): Microscopic Examination of H\&E stained sections of cases treated without concentrated growth factor (CGF) revealed numerous irregular newly formed bone trabeculae at the implant site. Entrapped osteocytes were noted inside these trabeculae, while osteoblasts rimmed their border and large marrow spaces dispersed in between. Dilated blood vessels and extravasated red blood cells were observed within the marrow spaces.

Fibrous tissue formation was demonstrated in all specimens and ranged from loose collagen fibers to dense collagen condensation. The extent of fibrous tissue varied between specimens, but was mainly limited to restricted areas surrounding blood vessels. All specimens were free from inflammatory cell infiltration. Fig 3

Group B: (test group) Microscopic examination of H\&E stained sections of cases treated with concentrated growth factor (CGF) revealed the

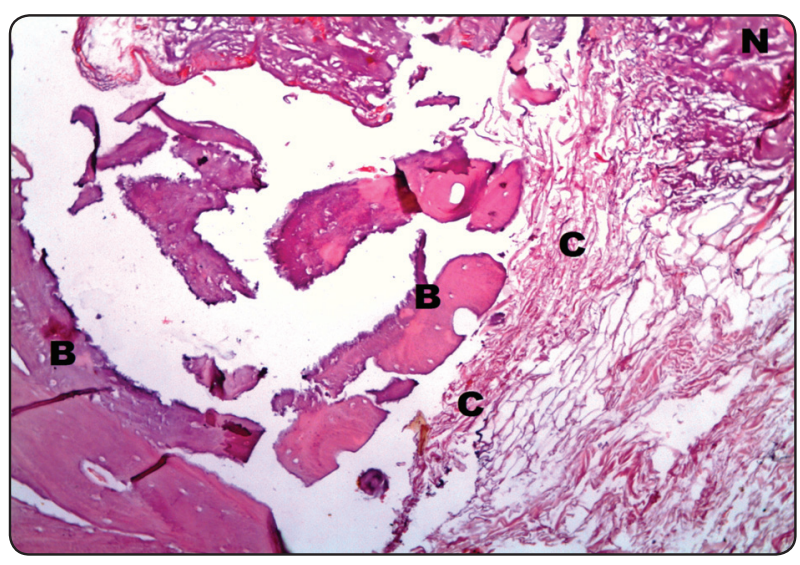

Fig. (3): Photomicrograph (H\&E x100) of group (A) 8 weeks post-operatively demonstrated regular arrangement of collagen fibers (C). Irregular trabeculae of newly formed woven bone $(\mathrm{N})$ and lamellar bone $(\mathrm{B})$ deposition of irregular newly formed bone trabeculae attempting to fill the area surrounding the implant site. Furthermore, osteoblastic rimming indicated their continuous deposition. Dilated blood vessels and extravasated red blood cells were observed in between the newly formed bone. Regular collagen deposition of variable densities was also detected. Fig 4

Histomorphometric analysis: $\mathrm{H} \& \mathrm{E}$ stained sections were assessed by ordinary light microscope to detect the newly formed bone trabeculae. An image analyzer computer system was used for generation of digital photomicrographs for histomorphometric analysis. The areas containing the most intense new bone formation were chosen for evaluation. These areas were masked by a blue binary colour to be measured by the computer system. Mean values were obtained for each specimen.

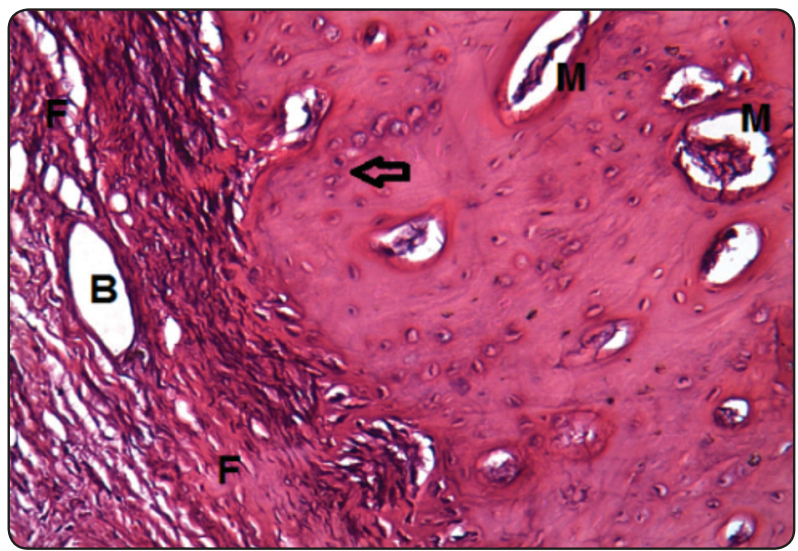

Fig. (4): Photomicrograph (H\& Ex 200) of Group (B) 8 weeks postoperatively demonstrating newly formed bone almost filling the defect. Viable osteocytes entrapped in lacunae (arrow), and marrow spaces (M) Organized collagen fibers (F) and dilated blood vessels (B) are seen at the boundaries of the defect

Histomorphometric evaluation: The percentage of the area occupied by the newly formed bone trabeculae was measured as area and area percent in a standard measuring frame in 10 fields in each group (A: treated without CGF, B: treated with CGF) after 8 weeks, using.> magnification (x400) 
by light microscopy transferred to the screen of the image analyser computer system.

In group A treated without CGF, the mean area percent was $25.662 \%$. It ranged from a minimum $6.42 \%$ of to a maximum of $42.81 \%$. While In group B treated with CGF, the mean area percent of newly formed bone was $66.939 \%$. It ranged from a minimum $56.71 \%$ of to a maximum of $91.87 \%$. Tab 1, Fig 5

Student's $\mathrm{t}$-test revealed that the difference between both groups was statistically significant $(\mathrm{p}=0.0001)$. Tab 2

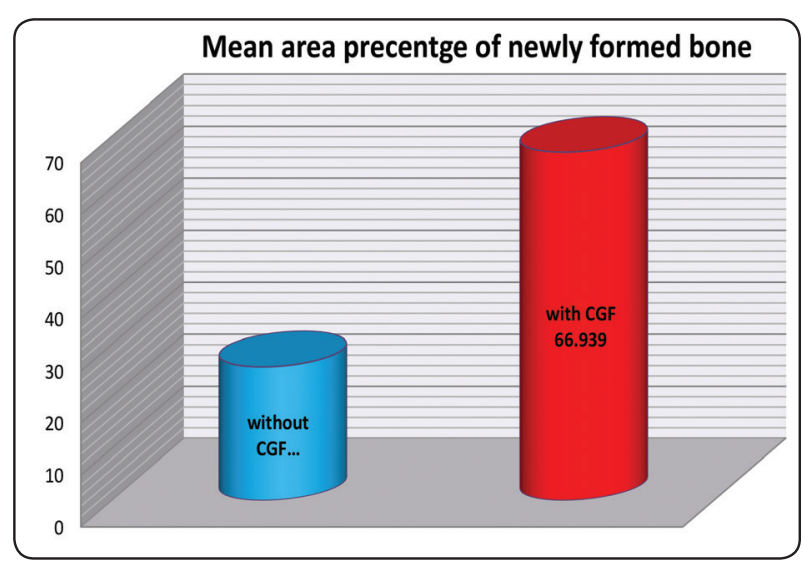

Fig. (5): Mean area percent of newly formed bone in both groups 8 weeks post operatively.

TABLE (1) Histomorphometric findings in both groups 8 weeks post operatively.

\begin{tabular}{|c|c|c|c|c|c|c|c|c|}
\hline & \multicolumn{4}{|c|}{ Group A (Without CGF) } & \multicolumn{4}{c|}{ Group B (With CGF) } \\
\hline Filed & Area & Area fraction & Area Fill & Area \% & Area & Area fraction & Area Fill & Area \% \\
\hline Total & 155440.6 & 2.58 & 2.95 & 256.62 & 838467.29 & 6.71 & 33.13 & 669.39 \\
\hline Mean & 15544.06 & 0.258 & 0.295 & 25.662 & 83846.729 & 0.671 & 3.313 & 66.939 \\
\hline std Dev & 5542.558 & 0.09899495 & 0.19092 & 9.751 & 2615.2132 & 0.056568542 & 0.6576 & 5.4235 \\
\hline std Erro & 2891.887 & 0.0480925 & 0.06343 & 4.773 & 43217.557 & 0.048909213 & 1.2233 & 4.8882 \\
\hline Max & 34542.12 & 0.57 & 0.73 & 56.71 & 471844 & 0.92 & 13.91 & 91.87 \\
\hline Min & 4531.64 & 0.06 & 0.07 & 6.42 & 25860.34 & 0.43 & 0.71 & 42.81 \\
\hline Range & 30010.48 & 0.51 & 0.66 & 50.29 & 445983.66 & 0.49 & 13.2 & 49.06 \\
\hline
\end{tabular}

TABLE (2) Statistical significance of the difference in the mean area percent of newly formed bone in both groups 8 weeks post operatively using Student's t test.

\begin{tabular}{|l|l|l|l|l|l|l|}
\hline Group & $\begin{array}{l}\text { Group A } \\
\text { Without CGF }\end{array}$ & $\begin{array}{l}\text { Group B } \\
\text { With CGF }\end{array}$ & T value & df & Std. Error Difference & P value \\
\hline Mean & 25.662 & 66.939 & 6.042 & 18 & 6.83 & $0.0001 * * *$ \\
\hline SD & 15.0936144 & 15.45777147 & & & & \\
\hline SEM & 4.773019962 & 4.88817654 & & & & \\
\hline N & 10 & 10 & & & & \\
\hline
\end{tabular}

$d f:$ degree of freedom, *** extremely statistically significant 


\section{DISCUSSION}

Osseointegration of dental implant is a determinant factor in the stability and long term success various strategies are being explored to improve the quality and accelerate the osseointegration of a dental implant one of which is the bioactive molecules as CGF that have proven to be valuable in increasing osteoblastic differentiation and healing acceleration.

The different centrifugation procedure used to produce CGF gives a fibrin network with superior properties than previous generations of blood products. It shows higher cohesion and fibrin tensile strength and stability. Which provides easy handling, manipulation and application to the surgical defect; moreover it degrades over a longer period of time which allows for sustained release of the entrapped growth factors with in the fibrin matrix. shown in a study of biological properties by Bernardi et al $2017^{(16)}$

This modified centrifugation process also provides a matrix not only superior in the physical properties but loaded with higher concentrations of growth factors which accelerates bone generation and increases new bone formation as described by

\section{Öncü et al $2017^{(14)}$ and Palermo etal 2019 ${ }^{(15)}$}

Furthermore the fact that these concentrates are autologous and do not need bovine thrombin all the biocompatibility issues are avoided and the growth factors are of high quality as described by Rodella et al $2011^{(17)}$ in a immunohistochemical study whom stated from their results that CGF is a big fibrin matrix of high density and rich in growth factors which have positive effect on stimulation of cellular proliferation, matrix remodeling and neoangiogenesis.

In the present study the potential effect of CGF on acceleration of osseointegration in immediate dental implants was investigated via histologically in animal model. Histomorphometric analysis showed significantly higher mean percentage of new bone formation in group B (treated with CGF) at $66.939 \%$, while mean percentage of new bone formation in group A (control group) was at $25.662 \%$

Student's $\mathrm{t}$-test revealed that the difference between both groups was statistically significant $(\mathrm{p}=0.0001)$. this indicated accelerated bone formation in group B which comes in agreement with results reported by Honda etal $2013{ }^{(7)}$, Durmuslar etal $2016^{(9)}$, Wang etal $2016^{(10)}$, Pirpir etal $2017^{(11)}$, Talaat etal $2018^{(13)}$; whom all found CGF to promote proliferation of osteoblasts and osteogenic maturation and mineralization which led to accelerated healing around the dental implant and further more implant stability and better surgical defect fill.

These results may be attributed to the fact that CGF stimulates cell growth proliferation and metabolic activity of fibroblasts, endothelial cells and osteoblasts which all are determinant cells in tissue healing and tissue regeneration which was confirmed in a study by Bonazza etal $2018^{(12)}$ whom investigated the positive effect of CGF on these critical 3 human cells in- vitro.

On the other hand, the control group A (control group) showed bone formation with numerous irregular newly formed bone trabeculae, entrapped osteocytes inside the trabeculae and osteoblasts rimming their border all which indicate the ongoing process around the implant which has been demonstrated by Mavrogenis et al $2009^{(4)}$ and Zupnik et al $2011^{\text {(3) }}$ confirming that once blood cells come into contact with implant surface it releases many cytokines and growth factors and differentiation factors further more form a fibrin matrix on the implant surface acting as an osteoconductive matrix promoting osseointegration all which are normal physiological cascade of events. 
The significantly higher mean percentage of new bone formation in group B -where the sockets were treated with CGF before placement of the immediate implants- may be attributed to the high concentration of growth factors present in this structure (CGF) and the sustained release of them due to the stronger more stable type of fibrin matrix produced also shown by Öncü etal $2016{ }^{(14)}$ whom described this modified centrifugation procedure which allows for production of fibrin network loaded with high concentration of growth factors than in other blood products which of course amplifies the quality and quantity of bone regeneration and tissue healing around the immediate implant. Furthermore demonstrated in studies by Sohn et al 2011 ${ }^{(5)}$ and Sacco et al $2016^{(6)}$ and Bernardi et al 2017 ${ }^{(16)}$.

\section{CONCLUSION}

Within the limitations of this study it may be concluded that application of CGF to the socket walls before placement of immediate implant may enhance the healing of bone surrounding implants and accelerate osseointegration offering a convenient and affordable choice for implant placement. Application of this method may, therefore, be effective in enhancing the rate of osseointegration and allow for early loading of immediate implants. Future clinical studies are needed to demonstrate the positive effect of CGF application in different fields of oral surgery

\section{REFERENCES}

1- American Academy of Periodontology : Parameter on Placement and Management of the Dental Implant. J Periodontal 2000 ;71 (5): 870-2.

2- Gokcen-Rohlig B, Yaltirik M, Ozer S, TuncerED, Evlioglu G. Survival and success of ITI implants and prostheses: retrospectivestudy of cases with 5-year follow-up. Eur J Dent 2009; 3: 42-49.

3- Zupnik J, Kim S-W, Ravens D, Karimbux N, Guze K. Factors associated with dental implant survival: a 4-year retrospective analysis. J Periodontol 2011; 82:1390-1395.
4- Mavrogenis A.F., Dimitriou R., Parvizi J., Babis G.C.. Biology of implant osseointegration. J Musculoskelete Neuronal interact. 2009;9(2):61-71.

5- Sohn DS, Heo JU, Kwak DE, Kim JM. Bone regeneration in maxillary sinus using an autologous fibrin-rich block with concentrated growth factors alone. Implant Dent .2011; 20 (5): 389-95

6- Sacco L , Park HC, Kim SG, Oh JS, You JS, Kim JS, Lim SC . Early bone formation at a femur defect using GF and PRF grafts in adult dogs: a comparative study. Implant dentistry. 2016; 25(3):387-93.

7- Honda H, Tamai N, Naka N, Yoshikawa H and Myoui A. bone tissue engineering with bone marrow- derived stromal cells integrated with concentrated growth factor in Rattus norvegicus calvaria defect defect modle. J Artif Organs. 2013;16(3):305-15.

8- Chen Y, Cai Z, Zheng D, Lin P, Cai Y, Hong S, Lai Y and $\mathrm{Wu} \mathrm{D}$. inlay osteotome sinus floor elevation with concentrated growth factor application and simultaneous short implant placement in severely atrophic maxilla. Sci Rep. 2016;6:1-8.

9- Durmuslar MC, Balli U, Dede FO, Misir AF, Baris E, Kurkcu M and Kahraman SN. Histological evaluation of the effect of concentrated growth factor on bone healing . J Craniofac Surg. 2016 ; 27(6):1494-7.

10- Wang F, Sun Y, He D and Wang L. effect of concentrated growth factors on the repair of goat Tempromandibular joint. J Maxillofac Surg. 2017;75(3):498-507.

11- Pirpir C, Yilmaz O, Candirli C and Balaban E. Evaluation of effectiveness of concentrated growth factor on osseointegration. Int J Implnt Dent. 2017; 3 (1): 7-12.

12- Bonazza V, Borsani E, Buffoli B, Parolini S, Inchingolo F, Rezzani R and Rodella LF. In vitro treatment with concentrated growth factors (CGF) and sodium orthosilicate positively affects cell renewal in three different human cell lines . Cell Biol Int.2018; 42(3): 353-64.

13- Talaat WM, Ghoneim MM, Salah O and Adly OA. Autologous bone marrow concentrates and concentrated growth factors accelerate regeneration after enucleation of mandibular pathologic lesions. J Craniofac Surg. 2018

14- Oncu E, Bayram B, Kantarci A, Gulsever S and Alaaddinoglu E. Positive effect of platelet rich fibrin on osseointegration . Med Oral Pathol Oral cir Bucal. 2017; 21 (5): 601-7 
15- Palermo A, Ferrante F, Stanca E, Damiano F, Gnoni A, Batani T, Demitri C, Siculella L. Release of VEGF from dental implant surface (IMI implant) coated with concentrated growth factors (CGF) and liquid phase of CGF ( LPCGF): Invitro results and future expectations. Appl. Sci. 2019; 9: 2114-21

16- Bernardi S, Mummolo S, Tecco S, Continenza M \& Marzo G. Histological characterization of Sacco's concentrated growth factors membrane. Int. J. Morphol. 2017; 35(1):114-119

17- Rodella L F, Favero G, Boninsegna R, Buffoli B, Labanca M, Scarì G, Sacco L, Batani T \& Rezzani R. Growth factors, CD34 positive cells, and fibrin network analysis in concentrated growth factors fraction. Microsc. Res. Tech., 74(8):772-7, 2011. 